\title{
(2) OPEN ACCESS \\ Multisystem organ failure secondary to acute generalised exanthematous pustulosis (AGEP) with atypical presentation resembling septic shock
}

\author{
Karen Cravero (10,' Teja Chakrala, ${ }^{1}$ Andrew Shychuk ${ }^{2,3}$
}

\begin{abstract}
${ }^{1}$ University of Florida College of Medicine, Gainesville, Florida, USA

${ }^{2}$ Internal Medicine, University of Florida College of Medicine, Gainesville, Florida, USA ${ }^{3}$ University of Florida, Gainesville, Florida, USA
\end{abstract}

\section{Correspondence to} Dr Andrew Shychuk; andrew.shychuk@medicine. ufl.edu

Accepted 21 January 2022

Check for updates

(c) BMJ Publishing Group Limited 2022. Re-use permitted under CC BY-NC. No commercial re-use. See rights and permissions. Published by BMJ.

To cite: Cravero K, Chakrala T, Shychuk A. BM Case Rep 2022:15:e247040. doi:10.1136/bcr-2021

247040

\section{SUMMARY}

A woman was admitted for sepsis secondary to cellulitis. After clinical improvement of sepsis, non-follicular small pustules were observed on the trunk, limbs and face while vesicles/bullae and skin exfoliation were noted on upper extremities. Larger systemic manifestations included fever, hypertension and tachycardia. Laboratory results revealed neutrophilic leukocytosis, eosinophilia, mild transaminitis and acute renal failure. Despite treatment for potential sepsis and discontinuation of offending agents, her condition worsened leading to haemodynamic instability and renal failure requiring vasopressor support, intubation and continuous venovenous haemodialysis. Skin biopsy revealed a diagnosis of acute generalised exanthematous pustulosis (AGEP), a rare condition usually caused by antibiotic treatment. The suspected offending drug was clindamycin, with possible combined effects by metronidazole and/or vancomycin. Improvement of skin manifestations were seen within 48 hours of starting systemic steroids. Here, we present an uncharacteristic case of AGEP clinically presenting with atypical skin lesions, severe systemic involvement mimicking septic shock, which culminated in multisystem organ failure.

\section{BACKGROUND}

Acute generalised exanthematous pustulosis (AGEP) is a rare severe cutaneous adverse reaction afflicting 1-5 patients per million per year. ${ }^{1}$ It is seen in both men and women, however, it tends to have a female predominance. A genetic component to susceptibility is suspected, as human leucocyte antigens B51, DR11 and DQ3 are found to be more commonly identified in patients with AGEP. ${ }^{1}$ Furthermore, Navarini et al described a higher percentage of IL36 receptor antagonist (IL36RN) mutations in AGEP patients with mucosal involvement. ${ }^{23}$

AGEP typically presents as an abrupt onset of superficial, pin-sized, sterile, non-follicular pustules usually on a background of erythematous and edematous skin. ${ }^{14}$ Pustules are often first identified in intertriginous areas, rapidly expanding to the trunk and limbs without involvement of the palms and soles. Systemic manifestations are usually limited to fever $\left(>38^{\circ} \mathrm{C}\right)$ and neutrophilia $\left(>7.5 \times 10^{9} / \mathrm{L}\right)$, while eosinophilia is seen in about $30 \%$ of all cases. ${ }^{5}$ Mucosal membrane involvement is absent or minimal, and if present, is usually localised to the lips. ${ }^{5}$ Rarely, AGEP cases may present with mild hepatic and kidney injury. ${ }^{6-8}$
AGEP is a type intravenous hypersensitivity reaction caused most commonly by antibiotics, although other drug classes, viral infections and unknown triggers have been reported. ${ }^{9}$ Onset of skin manifestation is highly variable, with clinical signs observed in less than 48 hours $^{8}$ or up to weeks. ${ }^{10}{ }^{11}$ In most cases, complete resolution of pustules and fever is achieved within 2 weeks following discontinuation of the offending agent, when skin lesions transition from pustules to diffuse exfoliation which may last for extended periods of time. ${ }^{15}$ The above described presentation is by far the most common way AGEP may manifest. Unusual cases have been reported in which AGEP may present with multiple vesicles, large bullae, and diffuse skin desquamation $^{12}$; a presentation more commonly observed with toxic epidermal necrolysis (TEN). TEN is a separate entity with its own unique manifestations and pathophysiology; however, atypical cases of AGEP clinically presenting with overlapping TEN features (appropriately named TEN-like AGEP) have been described in the literature. ${ }^{13} 14$ Presentation of TEN-like AGEP cases are often associated with mucous membrane involvement. They present with, skin manifestations observed in both conditions, however, demonstrate AGEP specific histopathology. ${ }^{13}$ While most of these cases tend to follow the clinical course of AGEP with full recovery, more severe events can present with signs resembling septic shock. These more severe forms may lead to the requirement of ICU-level care and vasopressor support. Multisystem organ failure may also occur with consequential increase in mortality rates. To the extent of the authors' knowledge, there have only been four cases reported of TENlike AGEP progressing to shock and multiple organ dysfunction. ${ }^{13-15}$

It is important to keep AGEP in the differential when patients develop a septic shock-like picture in association with skin manifestations of pustules and/or bullae shortly after drug administration. Given the rarity of AGEP, it is important for physicians to familiarise themselves with the spectrum of presentations to aid with timely diagnosis and therapeutic interventions. Herein, we describe a case of severe AGEP with overlapping TEN skin manifestation, which rapidly progressed into shock and multisystem organ failure.

\section{CASE PRESENTATION}

A morbidly obese African-American woman presented with acute cellulitis superimposed on 
chronic bilateral lower extremity lymphedema. At the time of admission, the patient stated that she had a history of stable lymphedema for over 3 years, but within the last week swelling in both legs increased, leading to severe pain, weakness and difficulty with ambulation. A day prior to presentation, she noticed white drainage from her left lower extremity prompting her to visit her local emergency department. She was found to be tachycardic, hypertensive, and febrile. Laboratory results were significant for an increased white cell count (WCC) $\left(31 \times 10^{9} / \mathrm{L}\right)$, mild liver dysfunction and acute renal failure. She was given acetaminophen, and intravenous fluids. Blood cultures were collected, and she was started on intravenous ceftriaxone. The patient was transferred to our hospital for further management of sepsis secondary to left lower extremity infection.

On presentation (day 1), she was hypertensive 199/73 mm $\mathrm{Hg}$, tachycardic (103 bpm), with temperature of $37.8 \mathrm{C}$ and saturating $98 \%$ on room air. On examination, she was alert and oriented to person, place and time. Her bilateral lower extremities were hyperpigmented, dry, scaly and thickened throughout. Significant bilateral pitting oedema was also present with circumferential induration. Numerous deep fissures, several containing maggots, were present in addition to purulent fluid leakage from the medial and plantar surfaces of both feet. Aside from severe pain and tenderness to palpation bilaterally, the patient denied any other symptoms. Initial labs were notable for an elevated lactic acid, creatinine and white WCC. No fluid collections or thromboses were present on imaging. Full infectious workup was ordered, and the patient was started on empiric antibiotic therapy with intravenous vancomycin, cefepime and metronidazole. On day 2, a one-time dosing of intravenous clindamycin was added for additional gram-positive coverage. Over the next 4 days, the patient's condition improved with resolution of her symptoms and haemodynamic instability. Her WCC steadily continued a downward trend to near-normal levels. Patient also reported decrease in pain in both legs.

On the late hours of day 5, approximately 87 hours after the first dose of clindamycin, the patient became febrile (37.6 C) and her WCC began to rise. Her mentation was normal, and she was deemed stable, not requiring an escalation of care. Repeat blood and urine cultures along with imaging studies were obtained, which revealed no new infections or abnormalities. During the early hours of day 6, patient developed another fever spike (38 C) with significant neutrophilia despite being on broad spectrum antibiotic coverage (intravenous vancomycin and cefepime). At this point, clindamycin and metronidazole were added back to the antibiotic regimen. At approximately 14:00 hours, the patient received a dose of clindamycin, the second one overall since being admitted.

Approximately 1 hour later, a skin lesion was first observed on her right medial thigh. Over the next few hours, her condition quickly deteriorated. She became unresponsive, haemodynamically unstable, which necessitated escalation of care and she was transferred to the intensive care unit for management of possible septic shock. Several hours later, the skin condition worsened. Physical examination revealed numerous scattered small studded pustules over an erythematous and edematous background, with intertriginous predominance. Small vesicles and flaccid bullae were also present in the upper and lower extremities as well as the neck region. Diffuse nonblanching redness was present throughout the body. Erythematous plaques with desquamation, denuded erosions and excoriations were also observed, most significantly under the breasts, and the posterior area of both ears (figure 1). There was no evidence of mucous membrane involvement. During
A

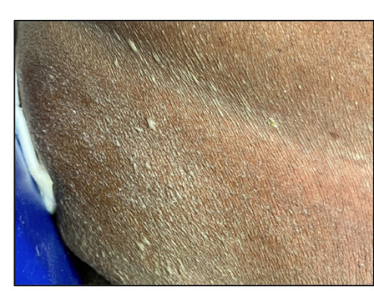

C

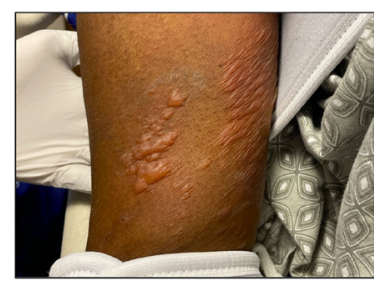

$\mathrm{E}$

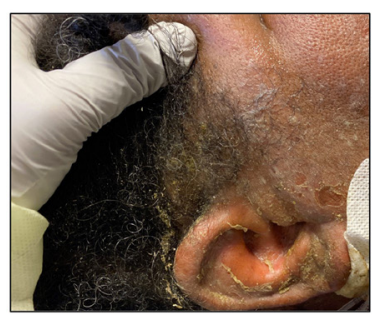

B

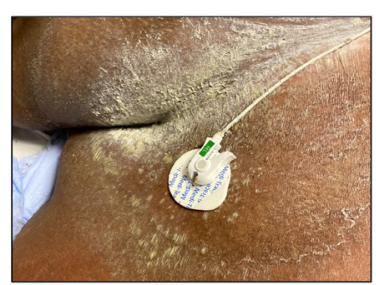

D

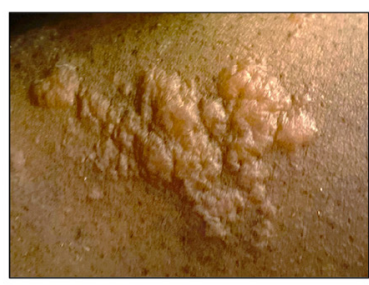

$\mathrm{F}$

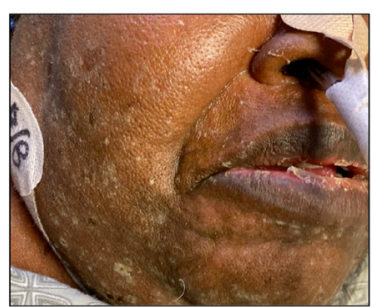

Figure 1 Clinical manifestations of AGEP: multiple, small, nonfollicular pustules present in $(A)$ inframammary folds and (B) right axilla. (C) Development of vesicles and bullae in upper extremities. (D) Increased magnification of vesicles/bullae identified. (E) Desquamation and erythematous skin (right ear) (F) Facial oedema and facial pustules. Similar lesions were also seen in torso, back and contralateral arm. AGEP, acute generalised exanthematous pustulosis.

this time, the patient had become encephalopathic requiring intubation for airway protection. Her urine output diminished, she developed metabolic acidosis and she was started on continuous veno-venous haemodialysis (CVVHD).

The next day, her cutaneous examination revealed more prominent pustules, vesicles, and superficial desquamation diffusely throughout her trunk and extremities (figure 2). Dermatology was consulted and a punch biopsy of affected skin was obtained. Clindamycin was stopped for suspicion of AGEP, and systemic corticosteroids was started. Laboratory studies revealed significant neutrophilic leukocytosis, mild eosinophilia, evidence of acute kidney injury, and some liver damage, with mild aspartate transaminase and alanine aminotransferase elevations. Because neutrophilia and eosinophilia are classic characteristics seen in AGEP, a clinical timeframe for WCC is presented in figure 3. Of note, absolute neutrophil levels obtained at time of rash detection, were the highest values to date. Levels of her kidney and liver function, are depicted in figure 4.

\section{INVESTIGATIONS}

Extensive laboratory testing was performed throughout the patient's hospital stay with relevant lab values at different time points of her treatment duration listed in table 1. CT imaging of the abdomen and pelvis revealed subcutaneous fat stranding; however, no soft-tissue gas was observed ruling out necrotising fasciitis. Chest imaging did not show any signs of new infections, no airspace opacities and no pleural effusion.

It is worth mentioning that the only positive blood culture results were obtained from medical records received from 


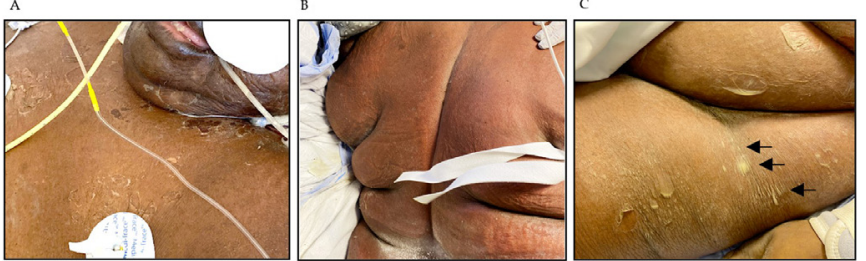

Figure 2 Second day of skin manifestations presented with more diffused and pronounced collarette-shaped desquamation, and erythematous skin in (A) chest, neck and (B) back areas. (C) Erosive lesions in the thigh and abdomen area with pustules coalescing into larger structures.

the institution the patient was originally treated at; which grew Streptococcus spp in both aerobic and anaerobic bottles. An extensive infectious workup performed at our hospital, including multiple blood and urine cultures, was largely negative. A skin punch biopsy was performed on a left proximal thigh lesion $(0.3 \mathrm{~cm} \times 0.5 \mathrm{~cm})$ and demonstrated collections of sub-corneal neutrophils overlying mild spongiosis, along with perivascular inflammation with infiltration of mostly neutrophils, but also eosinophils and lymphocytes to a lesser degree. Of special significance, no evidence of epidermal necrosis was noted. Figure 5 presents these histopathological findings.

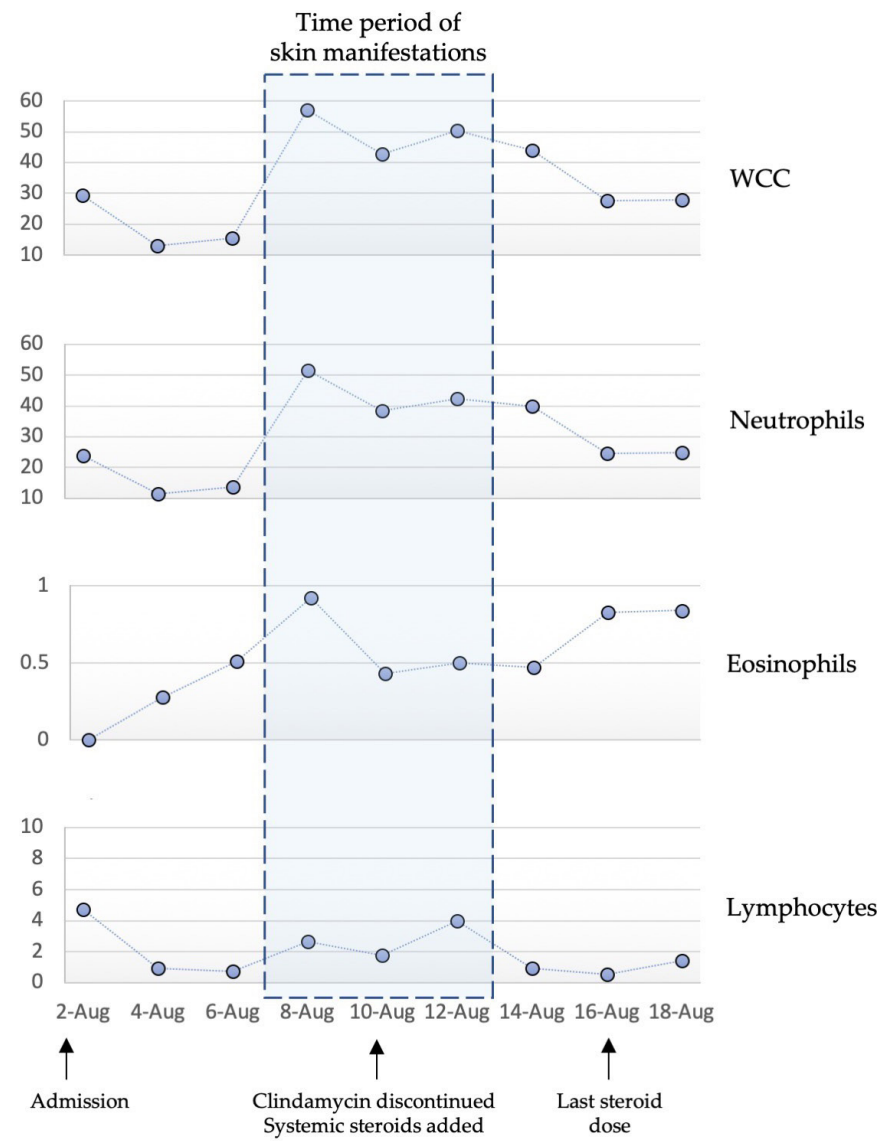

Figure 3 Absolute WCC at different times during length of clinical presentation. Counts demonstrated significant neutrophilia and mild eosinophilia during time of skin presentation. This figure was created by the authors specifically for inclusion in this manuscript. WCC, white cell count.

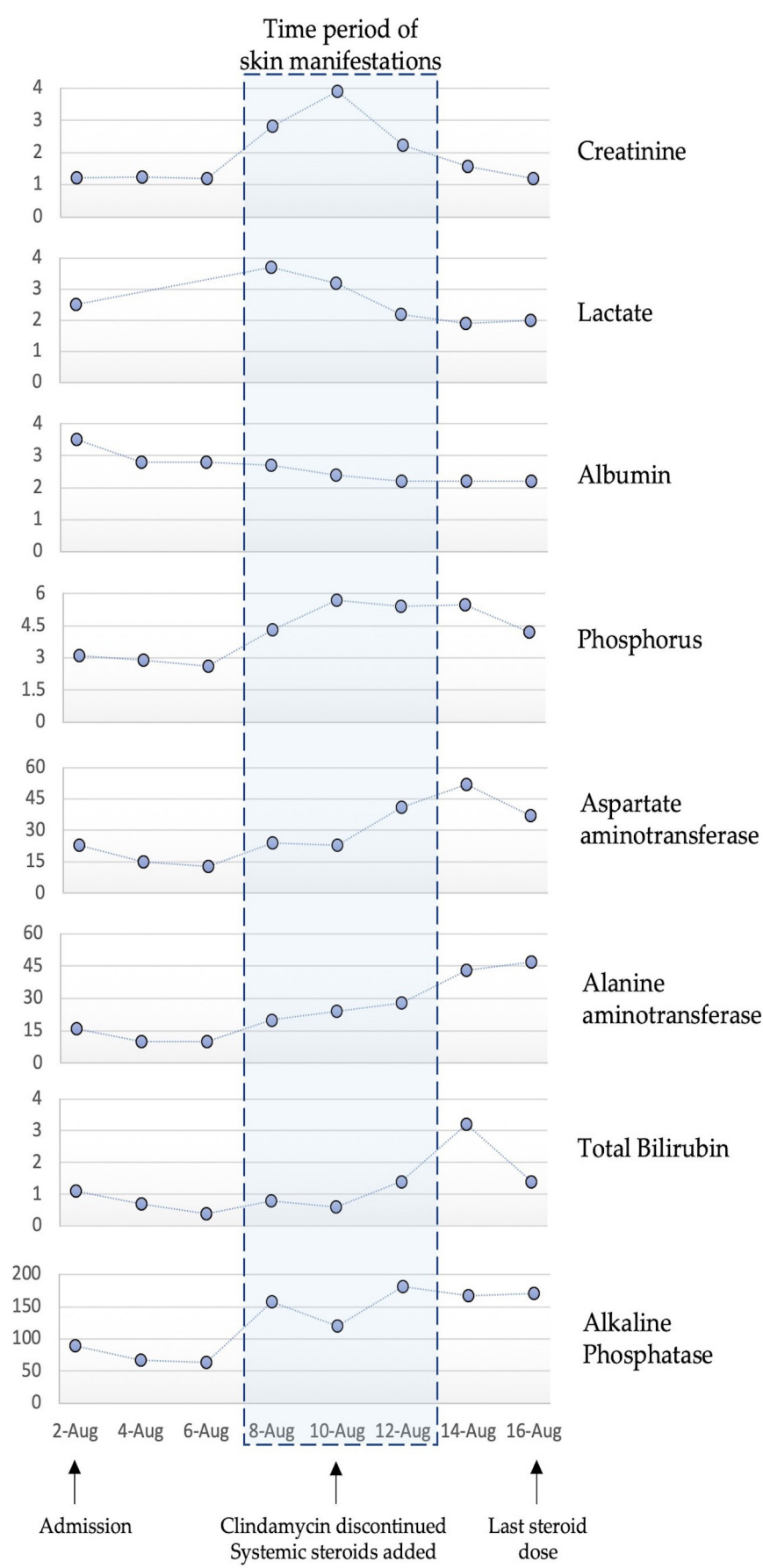

Figure 4 Pictorial representation of labs for kidney and liver function during length of clinical presentation. This figure was created by the authors specifically for inclusion in this manuscript.

\section{DIFFERENTIAL DIAGNOSIS}

The differential diagnosis for the case included AGEP, septic shock, pustular psoriasis, TEN, and Stevens-Johnson syndrome (SJS).

- A comprehensive infectious evaluation was negative. Culture of an open wound did grow Actinobacter iwoffii, a normal skin flora. Lack of an infectious agent along with the progressive deterioration of the patient's health despite broad antibiotic therapy, made the diagnosis of septic shock less likely.

Pustular psoriasis is very similar to AGEP in its presentation. ${ }^{16}$ AGEP has an acute onset, is a single episode, and 
Table 1 Relevant laboratory results across clinical timeline

\begin{tabular}{|c|c|c|c|c|c|c|}
\hline \multirow[b]{2}{*}{ Test } & \multirow[b]{2}{*}{ Reference range } & \multicolumn{5}{|l|}{ Results } \\
\hline & & Day 1 & Day 5 & Day 7 & Day 11 & Day 15 \\
\hline Leucocyte count $\times 10^{9} / \mathrm{L}$ & $1-7$ & 29.4 & 15.4 & 57.2 & 50.3 & 27.6 \\
\hline Eosinophil count $\times 10^{9} / \mathrm{L}$ & $0.03-0.46$ & 0 & 0.28 & 0.92 & 0.5 & 0.83 \\
\hline Lactate $\mathrm{mmol} / \mathrm{L}$ & $0.3-1.5$ & 2.5 & n.a. & 3.7 & 2.2 & 2 \\
\hline Albumin g/dL & $3.5-5.2$ & 3.5 & 2.8 & 2.7 & 2.2 & 2.3 \\
\hline Aspartate transaminase $\mathrm{U} / \mathrm{L}$ & $0-37$ & 23 & 15 & 24 & 41 & 37 \\
\hline Alanine transaminase U/L & $0-35$ & 16 & 10 & 20 & 28 & 47 \\
\hline Alk phos U/L & $33-133$ & 89 & 67 & 158 & 181 & 171 \\
\hline Total bilirubin $\mathrm{mg} / \mathrm{dL}$ & $0.0-1.0$ & 1.1 & 0.7 & 0.8 & 1.4 & 1.4 \\
\hline Creatinine $\mathrm{mg} / \mathrm{dL}$ & $0.38-1.02$ & 1.21 & 1.25 & 2.82 & 2.24 & 1.2 \\
\hline Comments & & Admission & Before rash & Rash detected & Rash improve & End of steroids \\
\hline
\end{tabular}

na, not applicable.

resolves within 2 weeks; whereas pustular psoriasis is a chronic condition with frequent flare ups and has a slower onset. ${ }^{17}$ Skin biopsy results in our patient lacked characteristic histological findings seen in psoriasis such as tortuous
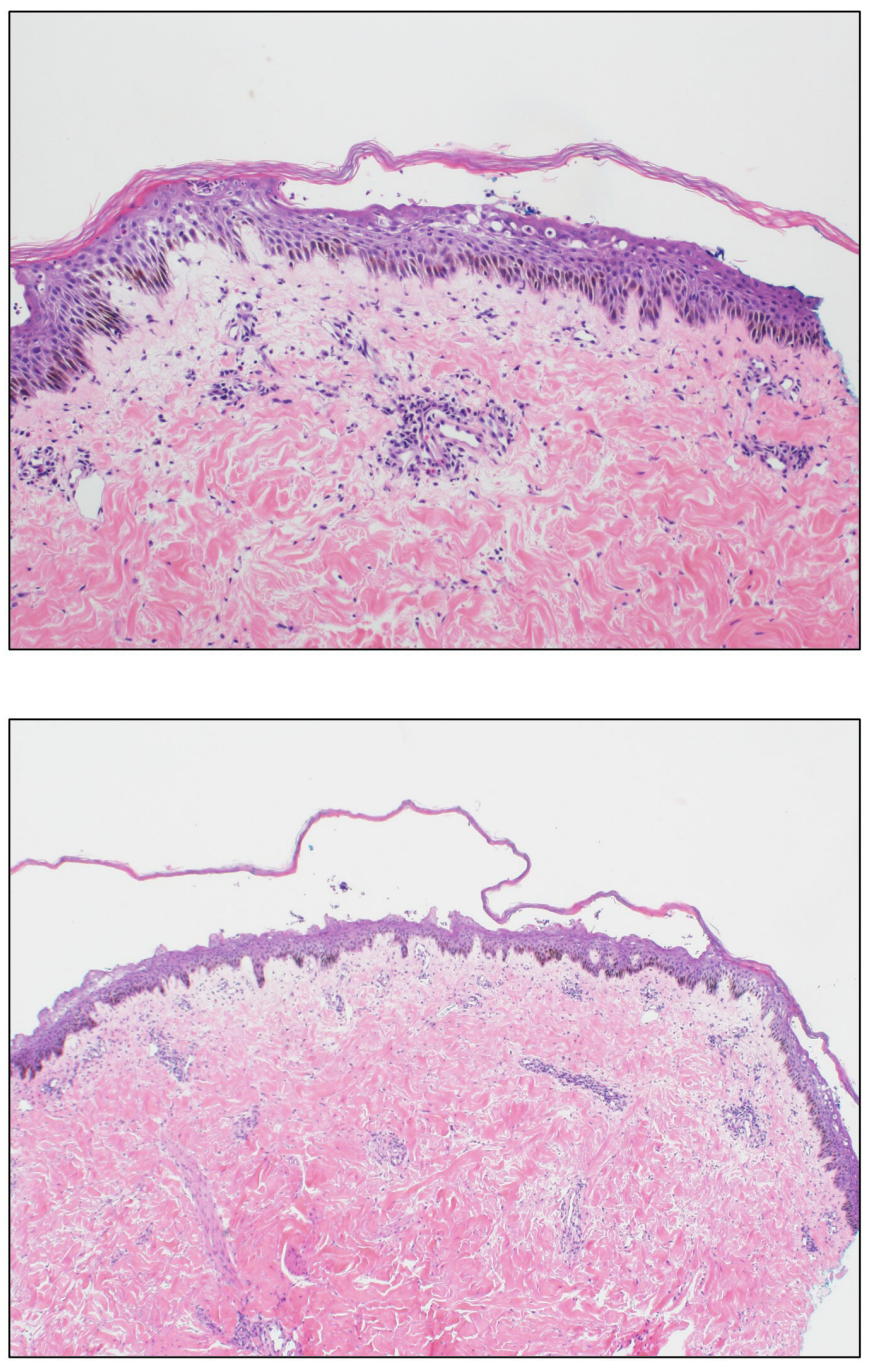

Figure 5 Histological sections of skin biopsy showing subcorneal pustulation and splitting. Epidermal spongiosis, perivascular inflammation, papillary dermal oedema and neutrophilic, eosinophilic and lymphocytic cell infiltrate. No evidence of epidermal necrosis. (H\&E stain). dilated blood vessels, psoriaform acanthosis, Munro's microabscesses and increased mitotic figures. ${ }^{17}$

- TEN and SJS tend to have mucous membrane involvement, a finding which was absent in our patient. The latency period between drug exposure and symptom onset is relatively more prolonged in these conditions. Drugs such as allopurinol and sulfonamides have been found to trigger SJS and TEN, but have not been associated with AGEP. ${ }^{12} 18$ Finally, both SJS and TEN are characterised by extensive epidermal necrosis. ${ }^{12}$ Our patient's physical signs resembled those seen in TEN, but her histological analysis was more suggestive of atypical TEN-like AGEP than TEN itself.

Overall, the temporal relationship of antibiotic initiation to skin eruption is strongly suggestive of drug reaction. When examining a patient with pustules or desquamation, the abovementioned diagnoses should be considered. In our case, given a high EuroSCAR score and the biopsy results, AGEP was the most likely diagnosis.

\section{TREATMENT}

Figure 6 is a pictorial representation of all antibiotics and systemic corticosteroids used with their respective time course. Treatments received by our patient at the outside hospital prior to transfer included acetaminophen, $3 \mathrm{~L}$ normal saline and $1 \mathrm{~g}$ of ceftriaxone. On day 1, after transfer to our hospital, the patient was empirically treated with intravenous metronidazole and cefepime for suspected sepsis. Intravenous vancomycin and one dose of clindamycin were added at later hours of the day. Due to the patient's clinical improvement, metronidazole was discontinued on day 4, while vancomycin and cefepime remained. During the late hours of day 5, early day six the patients' WCC and temperature began to rise, and on day 7 she was restarted on metronidazole and clindamycin. One dose of metronidazole was given in the early morning hours, while clindamycin was given in the early afternoon. About 1 hour post clindamycin (second dose overall), and approximately 5 hours after the last metronidazole treatment, skin manifestations were first detected. At this time, the patient became hypotensive, tachycardic with significant alteration in her mental status. Septic shock was suspected, and the patient received intravenous fluid resuscitation, along with vasopressor support for hypotension and continued with empiric broad-spectrum antibiotics (vancomycin, cefepime, clindamycin and metronidazole). Given her pressor requirement, patient's level of care was upgraded to ICU. She was sedated due to acute encephalopathy, intubated for mechanical ventilatory support and started on CVVH. Dermatology was consulted for 


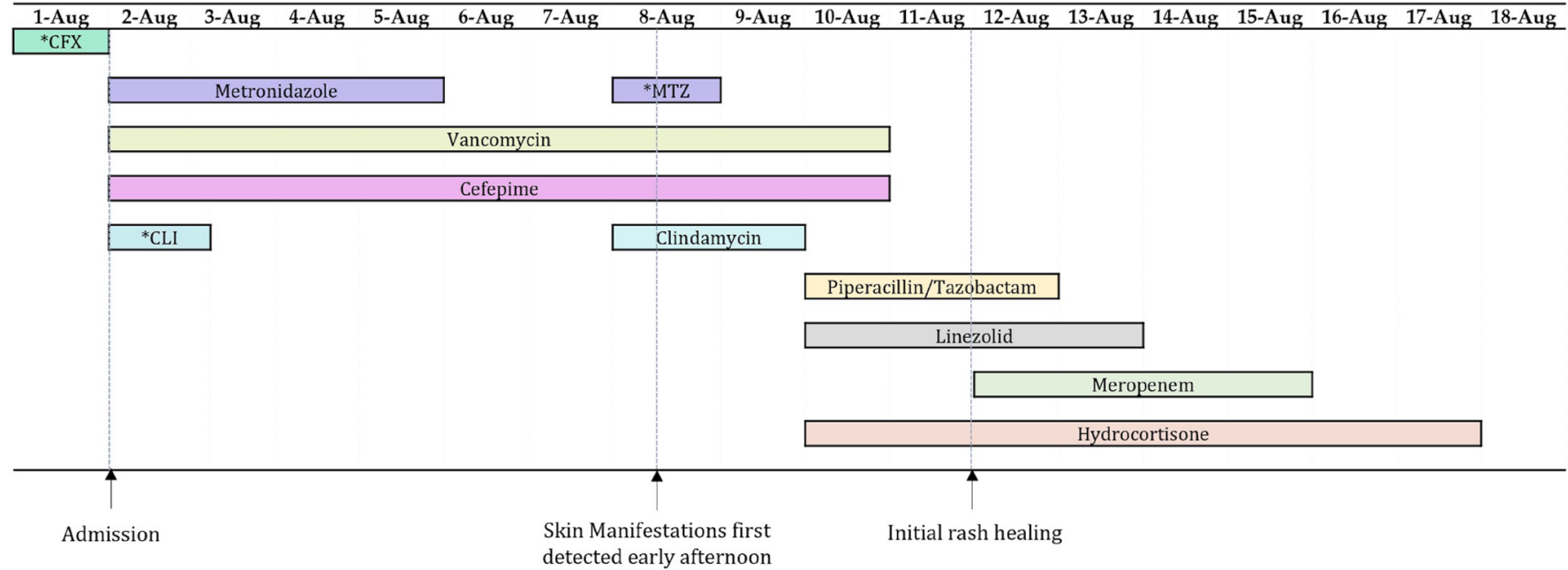

${ }^{*}$ CFX: Ceftriaxone

${ }^{*}$ CLI: Clindamycin

*MTZ: Metronidazole

Figure 6 Time period of antibiotic and steroid treatment in relation to the skin manifestations. This figure was created by the authors specifically for inclusion in this manuscript.

her cutaneous manifestations and per their recommendations pending biopsy results, clindamycin was discontinued (day 8). Despite use of topical corticosteroids (triamcinolone $0.1 \%$ ointment), the patient's skin eruption worsened over the next few hours, coinciding with rapid clinical decline, haemodynamic instability and multiorgan involvement with continued requirement of vasopressors.

By day 9, on AGEP confirmation via biopsy, cefepime and vancomycin were also discontinued due to the possibility of these agents contributing to the condition. Instead piperacillin/ tazobactam and linezolid were started for three and 4 days, respectively. Systemic corticosteroid therapy with hydrocortisone $50 \mathrm{mg}$ intravenous every 6 hours was also started, which led to a rapid clinical improvement of the skin manifestations within 24-48 hours post-treatment. Antibiotic therapy was transitioned to monotherapy with intravenous meropenem for 4 days after discontinuation of all other antibiotics. Corticosteroids were used for 8 days total, with the last dose given on day 15 of treatment.

\section{OUTCOME AND FOLLOW-UP}

Within 48 hours of clindamycin discontinuation and 24 hours after initiation of intravenous hydrocortisone, the rash improved. The patient's pustules and bullae progressed to extensive desquamation of her skin (figure 7). Systemic steroids were continued
A

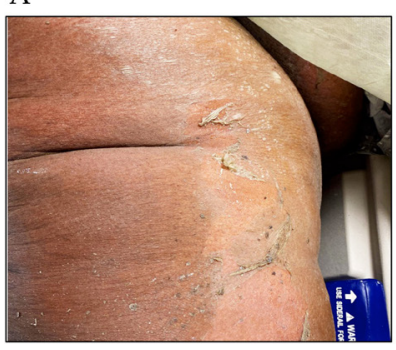

B

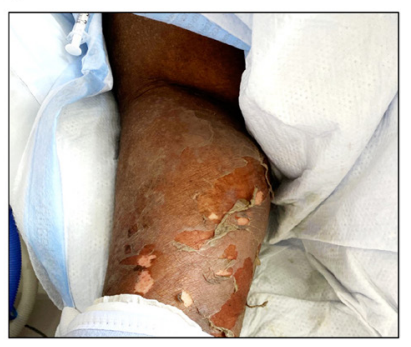

Figure 7 Resolution of pustules and vesicles with diffuse desquamation 2 days after discontinuation of drugs and steroid treatment. Representative pictures from areas in (A) abdomen and (B) right forearm. for an additional 5 days, and during this time the patient's antibiotics were discontinued sequentially with her remaining only on meropenem for the final 4 days of therapy. By day 14, vasopressors were no longer required, and the patient was no longer febrile. She exhibited spontaneous movement of her limbs and was responsive to tactile stimuli. On day 15 , the patient began responding to simple questions by moving her head; at this point steroids were discontinued. She remained intubated and continued to receive CVVHD for an additional 12 days. By the conclusion of this report, patient had been extubated, and started on intermittent haemodialysis. Both her WCC and liver enzymes were steadily improving; however, her serum creatinine levels and other markers of kidney function were worsening. Her mentation recovered, and to date she is conscious, alert and oriented, without any focal neurological deficits.

\section{DISCUSSION}

AGEP is a severe cutaneous adverse reaction (type intravenous hypersensitivity reaction) classically presenting with the acute eruption of hundreds of sterile pustules most commonly arising in intertriginous areas. Pustules are superimposed on a background of erythematous and edematous skin, with systemic manifestation limited to leukocytosis and fever. ${ }^{18}$ AGEP cases are traditionally self-limiting, resolving within 2 weeks of discontinuation of the causative agent with a mortality rate less than $5 \% .{ }^{19}$ Multiorgan involvement is rarely seen, although severe complications have been reported, especially with atypical presentation, such as the case presented here. Atypical AGEP refers to cases with superimposed manifestations that are not characteristically seen. In our patient, cutaneous findings also included vesicles, bullae, and extensive desquamation. Since these signs are usually associated with TEN, these uncharacteristic AGEP cases are referred to as TEN-like. As observed in our patient, these cases tend to be more severe in their presentation, with signs of septic shock, and may even lead to multiorgan failure. In the case described, our patient required intensive care treatment, vasopressor support and use of systemic corticosteroids. ${ }^{6} 1820$

While some AGEP cases have been attributed to viral infections, ${ }^{21}$ mercury contact ${ }^{22}$ and insect bites, the majority of cases (>90\%) result from medication exposure. ${ }^{59}$ The most common 


\begin{tabular}{lll}
\hline Table 2 & Drugs most commonly associated with AGEP development \\
\hline Drug class & Specific agents & References \\
\hline Antibiotics & $\begin{array}{l}\text { Ampicillin, amoxicillin, ceftriaxone, clindamycin, } \\
\text { vancomycin, pristinamycin and spiramycin }\end{array}$ & 148111823 \\
Antifungals & Nystatin, terbinafine, fluconazole & 2728 \\
\hline Anticonvulsants & Carbamazepine & 29 \\
Antimalarial & Hydroxychloroquine & 103031 \\
Antihypertensive & Diltiazem hydrochloride & 32 \\
\hline
\end{tabular}

culprits include antibiotics, antifungals and antihypertensives. Table 2 lists specific agents most frequently associated with AGEP development.

Because a patch test was not performed, the offending drug was unable to be identified. Nonetheless, based on the time period between administration of the drug to onset of symptoms ( $~ 80$ hours), and consequential improvement of skin manifestations on removal of the medication, we hypothesise that clindamycin was the causative agents. Clindamycin is a well described culprit of not only classical AGEP but also TEN-like incidents. ${ }^{4811} 132324$ Although we postulate that clindamycin is the offender, we cannot rule out the role that metronidazole, vancomycin and/or cefepime may have played on this patient's presentation. Interestingly, cases of AGEP presenting as septic shock, as seen in our patient, have been reported with the use of vancomycin. ${ }^{81823}$

Although a comprehensive description of the pathophysiology of AGEP is beyond the scope of this report, briefly, AGEP is categorised as a type intravenous hypersensitivity reaction mediated by $\mathrm{CD} 8+/ \mathrm{CD} 4+\mathrm{T}$ cells. ${ }^{25}$ During the initial phase, activated cytotoxic T-cells (referred in the literature as drug-specific T-cells), migrate to the skin and contribute to the development of sub-corneal vesicles by inducing keratinocyte apoptosis via granzyme B, perforin, and the Fas ligand mechanism. ${ }^{26}$ Infiltration of CD4 + Tcells leads to the release of CXCL-8, a potent neutrophil chemotaxis agent, causing subsequent recruitment of neutrophils and transformation of vesicles into pustules which are characteristic of AGEP. ${ }^{26}$ Other compounds released by drug-specific T-cells include interferon-gamma and granulocyte/ macrophage colony stimulating factor which further prolong the viability of neutrophils. ${ }^{2}$

Diagnosis of AGEP can be made by clinical assessment for which the EuroSCAR scoring system developed by Sidoroff $\mathrm{et} \mathrm{al}^{5}$ can be helpful. This system combines histological as well as clinical features to determine the likelihood of an AGEP diagnosis. Description of the rash, its distribution, timing, and presence of other symptoms are all taken into account. The algorithm yields a score between -10 to +12 , and its divided into 'no AGEP' (score -10-0), 'possible AGEP' (score 1-4); 'probable' (score 5-7) and 'definite' (score 8-12). Applying the AGEP validation score, our patient received a score of +12 , confirming the diagnosis. Table 3 shows the score allocated to our patient for each category. Clinical suspicion of AGEP can be validated by skin biopsy analysis.

Our case presents several unique factors. First, it is not a typical presentation of AGEP, involving signs that are usually seen in more severe conditions such as TEN. Furthermore, unlike most AGEP cases, our patient had multi-system involvement, with rapid deterioration, manifesting with hypotension, tachycardia, tachypnoea and fever; a picture resemblant of septic shock. Moreover, while discontinuing the suspected drug, and supportive care is the principal treatment, our case presents an instance in which treatment with systemic steroids
Table 3 AGEP validation score developed by EuroSCAR (European Severe Cutaneous Adverse Reactions) study group ${ }^{5}$

\begin{tabular}{|c|c|c|c|}
\hline Variable & $\begin{array}{l}\text { Our patient } \\
\text { results }\end{array}$ & Score & Comment \\
\hline \multicolumn{4}{|l|}{ Morphology } \\
\hline Pustules & Typical & +2 & Typical non-follicular sterile \\
\hline Erythema & Typical & +2 & Diffuse \\
\hline Distribution/pattern & Typical & +2 & Trunk, limbs, skin folds \\
\hline $\begin{array}{l}\text { Post-pustular } \\
\text { desquamation }\end{array}$ & Yes & +1 & Extensive \\
\hline \multicolumn{4}{|l|}{ Course } \\
\hline Mucosal involvement & No & 0 & \\
\hline Acute onset ( $\leq 10$ days) & Yes & 0 & \\
\hline $\begin{array}{l}\text { Resolution of pustules and } \\
\text { erythema } \leq 15 \text { days }\end{array}$ & Yes & 0 & \\
\hline Fever $\geq 38^{\circ} \mathrm{C}$ & Yes & +1 & \\
\hline $\begin{array}{l}\text { Polymorphonuclear } \\
\text { neutrophils }>7000 / \mathrm{mm}^{3}\end{array}$ & Yes & +1 & \\
\hline \multicolumn{4}{|l|}{ Histology } \\
\hline $\begin{array}{l}\text { Spongiform, subcorneal } \\
\text { pustule(s) with papillary } \\
\text { oedema, perivascular } \\
\text { infiltrates of neutrophils }\end{array}$ & Yes & +3 & \\
\hline Overall score $(-18$ to +12$)$ & & 12 & \\
\hline \multicolumn{4}{|c|}{$\begin{array}{l}\text { Overall score of }-10 \text { to } 0 \text { indicates no acute generalised exanthematous pustulosis } \\
\text { Score } 1-4 \text { 'Possible' } \\
\text { Score 5-7 'Probable' } \\
\text { Score 8-12 'Definite' }\end{array}$} \\
\hline
\end{tabular}

Giving the probability of AGEP diagnosis.

AGEP, acute generalised exanthematous pustulosis.

was warranted for clinical recovery. To the best of our knowledge, there have been only a few reported cases of AGEP with such severe presentation. It is crucial for physicians to become acquainted with the rarer AGEP manifestations so that recognition may lead to a faster, and accurate diagnosis thus facilitating a more appropriate treatment plan.

\section{Learning points}

Acute generalised exanthematous pustulosis (AGEP) is a rare, cutaneous drug reaction, classically presenting with small non-follicular pustules primarily visualised in intertriginous areas.

- AGEP can present with atypical skin manifestations mimicking TEN and should be suspected in patients being managed for septic shock associated with a negative infectious workup, along with a deteriorating clinical condition while on anti-infective agents.

- Most cases of AGEP are self-limited with good prognosis; however atypical TEN-like AGEP presentations can be more severe and lead to multiorgan failure.

Contributors $\mathrm{KC}$ contributed in the drafting, editing and revision of the case report. TC contributed in the editing and revision of the case report. AS contributed to the editing, revision and submission of the case report.

Funding The authors have not declared a specific grant for this research from any funding agency in the public, commercial or not-for-profit sectors.

Competing interests None declared.

Patient consent for publication Consent obtained directly from patient(s).

Provenance and peer review Not commissioned; externally peer reviewed.

Open access This is an open access article distributed in accordance with the Creative Commons Attribution Non Commercial (CC BY-NC 4.0) license, which permits others to distribute, remix, adapt, build upon this work non-commercially, 
and license their derivative works on different terms, provided the original work is properly cited and the use is non-commercial. See: http://creativecommons.org/ licenses/by-nc/4.0/.

Case reports provide a valuable learning resource for the scientific community and can indicate areas of interest for future research. They should not be used in isolation to guide treatment choices or public health policy.

\section{ORCID iD}

Karen Cravero http://orcid.org/0000-0001-7309-9425

\section{REFERENCES}

1 De A, Das S, Sarda A, et al. Acute generalised exanthematous pustulosis: an update. Indian J Dermatol 2018;63:22-9.

2 Szatkowski J, Schwartz RA. Acute generalized exanthematous pustulosis (AGEP): a review and update. J Am Acad Dermatol 2015:73:843-8.

3 Navarini AA, Simpson MA, Borradori L, et al. Homozygous Missense Mutation in IL36RN in Generalized Pustular Dermatosis With Intraoral Involvement Compatible With Both AGEP and Generalized Pustular Psoriasis. JAMA Dermatol 2015;151:452-3.

4 Aiempanakit K, Apinantriyo B. Clindamycin-induced acute generalized exanthematous pustulosis. Medicine 2020;99:e20389.

5 Sidoroff A, Dunant A, Viboud C, et al. Risk factors for acute generalized exanthematous pustulosis (AGEP) —results of a multinational case-control study (EuroSCAR). Br J Dermatol 2007;157:989-96.

6 Jakkidi M, Basmadjian C, Roy S. An illusion of septic shock: acute generalised exanthematous pustulosis with multiorgan dysfunction. BMJ Case Rep 2017:28:2017.

7 Sidoroff A. Acute generalized exanthematous pustulosis. Chem Immunol Allergy 2012;97:139-48.

8 Croy C, Buehrle K, Austin Szwak J. Clindamycin-associated acute generalized exanthematous pustulosis. J Clin Pharm Ther 2017;42:499-501.

9 Botelho LFF, Picosse FR, Padilha MH, et al. Acute generalized exanthematous pustulosis induced by cefepime: a case report. Case Rep Dermatol 2010;2:82-7.

10 Nili A, Zarei E, Ghamari A, et al. Acute generalized exanthematous pustulosis with a focus on hydroxychloroquine: a 10-year experience in a skin Hospital. Int Immunopharmacol 2020;89:107093.

11 Basnet S, Dhital R, Tharu B. Acute generalized exanthematous pustulosis: a rare side effect of clindamycin. J Community Hosp Intern Med Perspect 2019:9:285-6.

12 Huang S, Ahmed A, Hsu S, et al. Severe acute generalized exanthematous pustulosis with toxic epidermal necrolysis-like desquamation: a case series of 8 patients. JAAD Case Rep 2021;15:115-22.

13 Kostopoulos TC, Krishna SM, Brinster NK, et al. Acute generalized exanthematous pustulosis: atypical presentations and outcomes. J Eur Acad Dermato/ Venereol 2015;29:209-14.

14 van Hattem S, Beerthuizen GI, Kardaun SH. Severe flucloxacillin-induced acute generalized exanthematous pustulosis (AGEP), with toxic epidermal necrolysis (TEN)- like features: does overlap between AGEP and TEN exist? Clinical report and review of the literature. Br J Dermatol 2014;171:1539-45.

15 Krishna S, Ortega-Loayza A, Malakouti N, et al. A rapidly progressive and fatal case of atypical acute generalized exanthematous pustulosis. J Am Acad Dermatol 2014;71:e89-90.

16 Eyler JT, Squires S, Fraga GR, et al. Two cases of acute generalized exanthematous pustulosis related to oral terbinafine and an analysis of the clinical reaction pattern. Dermatol Online J 2012:18:5.

17 Kardaun SH, Kuiper H, Fidler V, et al. The histopathological spectrum of acute generalized exanthematous pustulosis (AGEP) and its differentiation from generalized pustular psoriasis. J Cutan Pathol 2010;37:1220-9.

18 Mawri S, Jain T, Shah J, et al. Vancomycin-induced acute generalized exanthematous pustulosis (AGEP) masquerading septic shock - an unusual presentation of a rare disease. J Intensive Care 2015;3:47.

19 Syed T, Abdullah AS, Mubasher M, et al. Acute generalized exanthematous pustulosis with multiple organ failure. Case Rep Dermatol 2021;13:47-53.

20 Lesterhuis WJ, Tjioe M, Stumpenhausen GA, et al. Acute generalised exanthematous pustulosis mimicking septic shock. Am J Med 2004;116:574-5.

21 Kaplan F, Topal E. Acute generalized exanthematous pustulosis due to Epstein-Barr virus infection in a neonate. Pediatr Dermatol 2021;38:1354-6.

22 Belhadjali H, Mandhouj S, Moussa A, et al. Mercury-induced acute generalized exanthematous pustulosis misdiagnosed as a drug-related case. Contact Dermatitis 2008;59:52-4.

23 Mohyuddin GR, Al Asad M, Scratchko L, et al. Acute generalized exanthematous pustulosis with multiple organ dysfunction syndrome. Am J Crit Care 2013;22:270-3.

24 Speeckaert MM, Speeckaert R, Lambert J, et al. Acute generalized exanthematous pustulosis: an overview of the clinical, immunological and diagnostic concepts. Eur J Dermatol 2010:20:425-33.

25 Feldmeyer L, Heidemeyer K, Yawalkar N. Acute generalized exanthematous pustulosis: pathogenesis, genetic background, clinical variants and therapy. Int J Mo/ Sci 2016;17:1214

26 Schmid S, Kuechler PC, Britschgi M, et al. Acute generalized exanthematous pustulosis: role of cytotoxic T cells in pustule formation. Am J Pathol 2002;161:2079-86.

27 Di Lernia V, Ricci C. Fluconazole-induced acute generalized exanthematous pustulosis. Indian J Dermato/ 2015:60:212.

28 Saliba E, Chrabieh R, Tannous Z. Fluconazole-induced acute generalized exanthematous pustulosis. Am J Emerg Med 2021;39:254.e5-54.e7.

29 Safa I, Ines L, Noureddine L, et al. Acute localized exanthematous pustulosis: clinical features, pathophysiology, and therapy. Dermatol Ther 2021;34:e15087.

30 Mercogliano C, Khan M, Lin C, et al. AGEP overlap induced by hydroxychloroquine: a case report and literature review. J Community Hosp Intern Med Perspect 2018;8:360-2

31 Reap LE, Rodd C, Larios J, et al. Hydrochlorothizide-induced acute generalised exanthematous pustulosis presenting with bilateral periorbital impetigo. BMJ Case Rep 2019;12:bcr-2017-223528.

32 Kim H-J, Jung K-D, Lee K-T, et al. Acute generalized exanthematous pustulosis caused by diltiazem. Ann Dermatol 2011;23:108-10.

Copyright 2022 BMJ Publishing Group. All rights reserved. For permission to reuse any of this content visit

https://www.bmj.com/company/products-services/rights-and-licensing/permissions/

BMJ Case Report Fellows may re-use this article for personal use and teaching without any further permission.

Become a Fellow of BMJ Case Reports today and you can:

Submit as many cases as you like

- Enjoy fast sympathetic peer review and rapid publication of accepted articles

Access all the published articles

Re-use any of the published material for personal use and teaching without further permission

Customer Service

If you have any further queries about your subscription, please contact our customer services team on +44 (0) 2071111105 or via email at support@bmj.com.

Visit casereports.bmj.com for more articles like this and to become a Fellow 